\title{
Determining Flow Propagation Direction from In-Flight Array Surface Pressure Fluctuation Data
}

\author{
Stefan Haxter* and Tobias Berkefeld ${ }^{\dagger}$ and Carsten Spehr ${ }^{\ddagger}$ \\ German Aerospace Center (DLR), 37073 Göttingen, Germany
}

$\begin{array}{ll} & \\ b & \text { wavenumber dirty map } \\ e & \text { steering vector } \\ f & \text { frequency } \\ H & \text { hermitian } \\ i & \text { imaginary unit } \\ k_{c} & \text { convective wavenumber } \\ k_{x}, k_{y} & \text { wavenumber } \\ L & \text { window size } \\ l_{x}, l_{y} & \text { coherence lengths } \\ M & \text { number of signal averages } \\ N & \text { number of transducers } \\ R & \text { cross-spectral matrix } \\ s, \hat{s} & \text { inclination factors } \\ u_{\varphi} & \text { phase velocity } \\ x, y & \text { transducer position } \\ & \end{array}$

\section{Nomenclature}
$\alpha \quad$ tilt angle of the coherence pattern
$\hat{\alpha} \quad$ tilt angle of the convective ridge
$\beta \quad$ angle to the center of the convective ridge
$\gamma \quad$ coherence
$\phi \quad$ spectrum
$\xi, \eta \quad$ transducer separation
$\hat{\xi}, \hat{\eta} \quad$ rotated transducer separation
$\xi,^{\prime} \eta^{\prime}$ rotated transducer separation

Subscript

$k \quad$ averaging window index

$n, m$ transducer indices

$x \quad$ length direction

$y \quad$ cross direction

\section{Introduction}

Knowledge about the characteristics of pressure fluctuations are important for predicting the aero-vibro acoustic excitation of surface panels exposed to a boundary layer flow. One of these characteristic features is the direction where the flow is propagating locally on the surface, especially when the coherence length is to be determined in flow direction. ${ }^{1}$ In the present paper some discrepancies in the resulting flow direction are discussed which were found when using different evaluation methods. Three methods of determining the "flow" angle from the data itself will be presented and the results will be compared. As it will be found, the use of the terms "phase velocity" and "convection velocity" have to be chosen carefully when discussing the propagation of pressure fluctuations on a surface as they can differ.

When using data wind tunnel experiments, the flow direction is known. In flight testing however, some effort has to be taken to determine the main direction of propagation. Rizzi et. $\mathrm{al}^{2}$ used an agile cone mounted on the outside of the airplane to indicate the local flow direction, which was then recorded by a video camera. Palumbo ${ }^{3}$ used a similar technique where the pitch of the aircraft was aligned so that the flow direction - indicated by a string attached to the outside of a window - would match a line imprinted on the window thereby indicating the alignment of the flow with an transducer array in an adjacent window of the airplane. Haxter\&Spehr ${ }^{4}$ used the coherence data from an in-flight array to determine the flow direction directly from the measured data by means of fitting a line through the positions above a threshold.

\footnotetext{
*Research Scientist, Department of Experimental Methods, stefan.haxter@dlr.de, No Member.

$\dagger$ Research Scientist, Department of Fluid Systems, No member

${ }^{\ddagger}$ Research Scientist, Department of Experimental Methods
} 
Pressure fluctuations underneath the turbulent boundary layer are commonly evaluated in the spatial domain (as performed by Palumbo ${ }^{3}$ and by Haxter\&Spehr ${ }^{4}$ ) and in the wavenumber domain (as performed by Abraham $^{5}$ and by Haxter et al. ${ }^{6}$ and summarized by Bull ${ }^{7}$ ). A conclusions about the flow angle can be drawn from each representation. In the wavenumber domain both, the inclination of the convective ridge and the position of the center of the convective ridge yield information about the flow direction. In the spatial domain, it is the tilt of the the coherence pattern that provides information hereof. In the following paper these three analysis methods for the determination of the flow angle are applied to the same dataset and their results compared. Any differences in flow angle resulting from the different methods will be subject to discussion.

It is expected that the methods yield the same results as no contrary observations were found in the literature. However it is foreclosed that the angles do not match in the specific case under consideration. The aim of the paper is to introduce the findings and to yield a first interpretation of a possible cause.

The paper will first give an overview of the flight test experiment evaluated. Secondly, the analysis methods are presented and the results presented afterwards. A first discussion of the findings will be given at the end and an assumption is made of what event in the boundary layer might cause the findings presented.

\section{Experiment}

Experiments were conducted on the DLR's Advanced Technology Research Aircraft 'ATRA'. The test carrier was equipped with three dummy windows in the vicinity of the wing in which a total of 30 pressure transducers were installed as an array. 30 relative pressure transducers of type Kulite XCL-093 with a pressure range of 5 PSI were installed. A quasi-random distribution was chosen in which each array position had a neighbor element in both, length and cross direction. In both directions, the spacings were chosen to increase logarithmically. The reference pressure was taken from a pinhole on the center dummy window and routed via a long narrow tube and a manifold to all transducers. The absolute reference pressure was measured using a SETRA model ASM1 wih a pressure renage of 0 to 16 PSI. For recording of the signals a Dewetron DEWE-818 data acquisition system with DEWETRON DEWE-51-PCI-128 A/D-frontends was used at a sampling rate of $50 \mathrm{kHz}$. Electrical noise from the airborne power supply system at a frequency of $400 \mathrm{~Hz}$ was present on the signals and was filtered out in the time domain using multiple narrow-band notch filters for the fundamental and the higher harmonics. The filter was applied twice, once on the normal signal and again on the reversed result from the first filtering in order to eliminate phase shifts resulting from the filtering. The flight condition was chosen as a typical cruise flight scenario ${ }^{1}$ and a total time of $600 \mathrm{~s}$ was recorded.

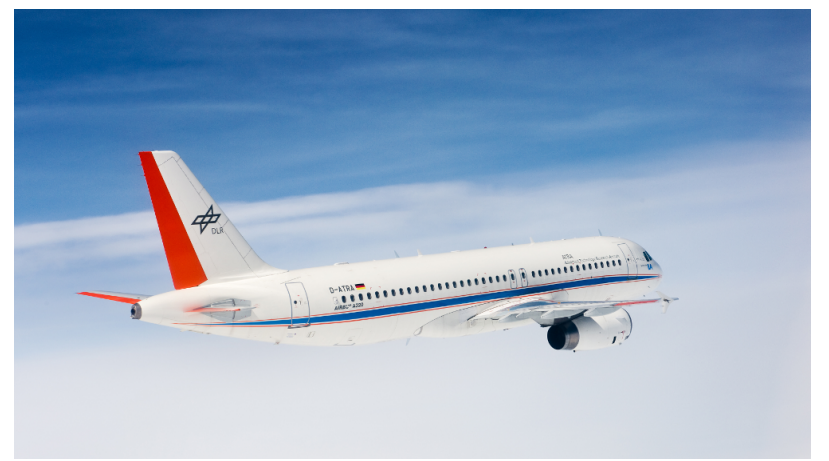

Figure 1. Test carrier D-ATRA (Advanced Technology Research Aircraft). Source: DLR CC-BY 3.0

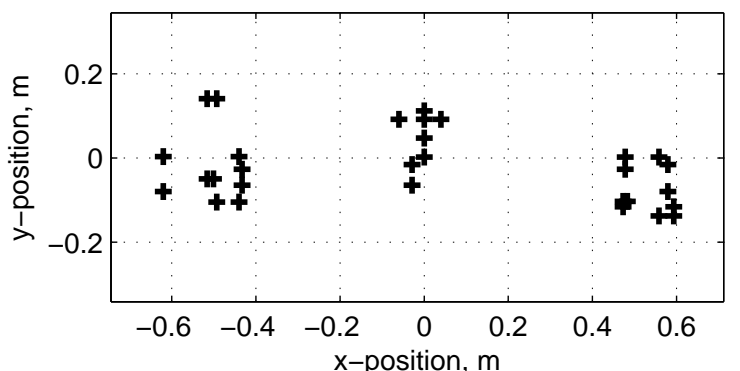

Figure 2. Sketch of the array positions installed on the test carrier.

\section{Signal Processing}

Data from the pressure transducer array were processed in both the spatial domain and the wavenumber domain. Prior to any evaluation, the cross-spectral matrix $R$ of the data was generated using Welch's method for estimating the cross-spectra. 


$$
R_{n m}=\sum_{k=1}^{M}\left(\phi_{n}^{k}\right) \cdot\left(\phi_{m}^{k}\right)^{H}
$$

where $H$ indicates the Hermitian and $\phi_{n}^{k}$ is the Fourier transform of the $k$-th window of the singnal of transducer $n$. A window size of $L=4096$ samples was used in order to ensure both, a complete propagation of turbulent structures over the entire length of the array. The total number of $k=14582$ averages was achieved using an overlap factor of $r=0.5$. Each window was weighted using a Hanning window function. The procedure results in a matrix $R$ for every frequency bin of the Welch estimation, so $R \equiv R(f)$.

In order to find the flow direction, three methods are proposed here. The first one is performed in the spatial domain and analyzes the coherence. The second approach is performed in the wavenumber domain and analyzes the inclination and the center position of the convective ridge.

\section{III.A. Spatial Domain Approach}

Each element $R_{n m}$ of the cross-spectral matrix can be normalized with the diagonal elements $R_{n n}$ and $R_{m m}$ to obtain the coherence $\gamma^{2}$.

$$
\gamma_{n m}^{2}=\frac{\left|R_{n m}\right|^{2}}{R_{n n} \cdot R_{m m}}
$$

Note that the magnitude of the cross-spectral entry is taken in the numerator of equation (2) thus discarding the phase information contained in $R_{n m}$. In order to find the flow direction, the spacing information between each transducer pair in array length direction $\xi$ and in array cross direction $\eta$ is required.

$$
\begin{gathered}
\xi_{n m}=x_{n}-x_{m} \\
\eta_{n m}=y_{n}-y_{m}
\end{gathered}
$$

A threshold value is applied to the square root of the coherence, $\gamma$. The threshold chosen here is $\gamma_{\text {thresh }}=$ $\exp (-1) \approx 36.7 \%$. The amplitude of the remaining points is neglected and just their position is processed further. A linear function is then fitted through the remaining pairs of $\xi(\gamma>\exp (-1))$ and $\eta(\gamma>\exp (-1))$ and results in the deviation of the flow angle from the array length axis. This analysis is governed by the largest separations of transducer spacings.

$$
\eta(\gamma>\exp (-1))=s \cdot \xi(\gamma>\exp (-1))
$$

The inclination factor $s$ is transformed into a flow angle $\alpha$ relative to the array length axis via

$$
\alpha=\operatorname{atan}(s)
$$

\section{III.B. Wavenumber Domain Approach}

As stated above, the phase content of the cross-spectral entries $R_{n m}$ are discarded when calculating the coherence in equation (2). When performing a wavenumber analysis on the data, this phase information is evaluated as well as the amplitude information contained in $R_{n m}$. The transformation is performed using a beamforming approach as used by Ehrenfried\&Koop. ${ }^{8}$

$$
b\left(k_{x}, k_{y}, f\right)=\frac{e\left(k_{x}, k_{y}\right) \cdot R(f) \cdot e\left(k_{x}, k_{y}\right)}{N^{2}}
$$

The resulting beamforming map $b$ is a representation of the pressure fluctuations in the wavenumber domain. The wavenumber positions $k_{x}$ and $k_{y}$ are arbitrarily chosen to fit the desired range of wavenumbers one whishes to evaluate. When all $k_{y}$ are chosen to be zero, the map results in a line and represents a onedimensional spectrum. As the flow is unlikely to be perfectly aligned with the array in a flight test scenario, the wavenumber grid is generated using $k_{y} \neq 0$. The steering vector $e$ is a planar wave propagation model generated via

$$
e\left(k_{x}, k_{y}\right)=\exp \left(-1 i \cdot\left(k_{x} x+k_{y} y\right)\right)
$$


where $x$ and $y$ are vectors containing all instances of $x_{n m}$ and $y_{n m}$ and $i=\sqrt{-1}$.

The beamform map is yet contaminated with array properties impressed on the result. A deconvolution procedure is required to remove the array's inherent point spread function from $b$ in order to obtain the desired underlying source map $q$. In the current evaluation DAMAS2.1 was chosen. ${ }^{9}$ Basic characteristics are however already visible in the "dirty" map $b$. Bull ${ }^{7}$ summarized this for the one-dimensional case, which are also valid for the two-dimensional case. The deconvolution results in the estimated source map $q$ whereat

$$
b=p * q
$$

with $p$ being the array's point spread function.

In boundary layer flows, the convective ridge is a very dominant feature of the wavenumber spectrum. It appears as a quasi-rhombic shape elongated in cross-stream direction. Its center position is located where the convective wavenumber $k_{c}$ is located. For subsonic flows, $k_{c}>k_{0}$ applies, where $k_{0}$ is the acoustic wavenumber.

In the analysis, the center of the convective ridge is determined by using the centroid of the $k_{x}$ and $k_{y}$ values attributed to the convective ridge. The position of this centroid is referred to as the convective wavenumber $k_{c}$. The attributed region was defined as every point lying no further than $-5 \mathrm{~dB}$ below the peak of the convective ridge. This method was considered reasonable for compensating noise-induced amplitude variations in the convective ridge which would occur when only taking the position of the maximum value.

The convective wavenumber represents the phase velocity of the pressure fluctuations.

$$
u_{\varphi}=\frac{f}{k_{c}}
$$

Note that the wavenumber domain is set up using units of inverse length $\left[\mathrm{m}^{-1}\right]$ instead of circular wavenumber of unit $\left[\mathrm{rad} \mathrm{m}^{-1}\right]$. The propagation direction of the phase of pressure fluctuations on the surface is obtained via:

$$
\beta=\operatorname{atan}\left(\frac{k_{y}}{k_{x}}\right)
$$

The inclination of the convective ridge is determined similar to the inclination of the coherence pattern in equation (5).

$$
k_{y}=\hat{s} \cdot k_{x}
$$

Since the dominant axis of the convective ridge is inclined $\pi / 2$ relative to the flow direction, $\alpha$ is determined via:

$$
\hat{\alpha}=\operatorname{atan}(\hat{s})+p i / 2
$$

The shape of the coherence pattern in the spatial domain and the shape of the convective ridge are intertwined. Both, a rhombic and an elliptic combination of longitudinal and lateral coherence length have been tested on experimental data by Haxter\&Spehr ${ }^{4}$ and the residual of both fit functions was similar. The rhombic shape however is easily transformable from the spatial to the wavenumber domain analytically, which is why many boundary layer surface excitation models use this fit. (Ref. 10-12). Regardless of the combination of the coherence length, the quasi-exponential drop of coherence of the pressure signals over distance can be interpreted as a Poisson window. ${ }^{13}$

The size of the coherence pattern in the spatial domain and the size of the convective ridge in the wavenumber domain are inversely proportional.

The coherence pattern is not dependent on the phase, as the phase is neglected in the determination of coherence in equation (2). The coherence pattern is believed to be connected with the flow direction of the boundary layer. ${ }^{1}$ It is tilted by an angle $\alpha$ relative to the array coordinate system. The angle of the coherence pattern will also be directly connected to the inclination of the convective ridge in the wavenumber domain due to the properties of the two-dimensional Fourier transform [14, p.168]. The direction retrieved from these two analyses therefore govern the direction of what will be called convection velocity in the following. At last, the centroid of the convective ridge results from the propagation of pressure phase on the surface and therefore the velocity resulting from the centroid position will be called phase velocity in the following. 


\section{Modeling Coherence Pattern and Wavenumber Spectra}

The coherence pattern was modeled combining the longitudinal and lateral coherence drop via multiplication as has been used by Corcos $^{10}$ and Efimtsov. ${ }^{11}$

$$
\tilde{\gamma}_{\text {model }}(\tilde{\xi}, \tilde{\eta}, f)=\exp \left(-\frac{|\tilde{\xi}|}{l_{x}(f)}-\frac{|\tilde{\eta}|}{l_{y}(f)}\right)
$$

The values $\tilde{\xi}$ and $\tilde{\eta}$ already incorporate the angle of flow direction $\alpha$ via

$$
\begin{gathered}
\tilde{\xi}=\cos \alpha \cdot \xi+\sin \alpha \cdot \eta \\
\tilde{\eta}=-\sin \alpha \cdot \xi+\cos \alpha \cdot \eta
\end{gathered}
$$

Equation (14) can therefore be written as:

$$
\gamma_{\text {model }}(\xi, \eta, \alpha, f)=\exp \left(-\frac{|\cos \alpha \cdot \xi+\sin \alpha \cdot \eta|}{l_{x}(f)}-\frac{|-\sin \alpha \cdot \xi+\cos \alpha \cdot \eta|}{l_{y}(f)}\right) .
$$

The normalized cross-spectral model including phase propagation is obtained by combining equation (14) with a complex propagation term $\tilde{\varphi}$.

$$
\tilde{\varphi}_{\text {model }}(\xi, f)=\exp \left(k_{\varphi}(f) \xi^{\prime} i\right)
$$

The propagation wavenumber $k_{\varphi}$ is determined from the phase velocity $u_{\varphi}$ via

$$
k_{\varphi}(f)=\exp \left(-\frac{2 \pi f}{u_{\varphi}(f)}\right) .
$$

Note that there is $\xi^{\prime}$ used instead of $\xi$ in the propagation term. The apostrophe indicates yet another, similar coordinate transform given by

$$
\xi^{\prime}=\cos \beta \cdot \xi+\sin \beta \cdot \eta
$$

so that

$$
\varphi_{\text {model }}(\xi, \eta, \beta, f)=\exp \left(-\frac{2 \pi(\cos \beta \cdot \xi+\sin \beta \cdot \eta) i}{u_{\varphi}(f)}\right) .
$$

The normalized cross-spectral model then is:

$$
\phi(\xi, \eta, \alpha, \beta, f)=\gamma(\xi, \eta, \alpha, f) \cdot \varphi(\xi, \eta, \beta, f)
$$

If the inclination of the coherence pattern direction and the phase propagation direction are equal, then $\xi^{\prime} \equiv \tilde{\xi}$. However, now these two angles can be used to set up different directions between the coherence distribution and the phase propagation. In the wavenumber domain, equation (22) yields

$$
q_{\text {modeled }}\left(k_{x}, k_{y}, \alpha, \beta, f\right)=\frac{1}{2 \pi} \int_{-\infty}^{+\infty} \int_{-\infty}^{+\infty} \gamma(\xi, \eta, \alpha, f) \cdot \varphi(\xi, \eta, \beta, f) \cdot \exp \left(i k_{x} \xi\right) \cdot \exp \left(i k_{y} \eta\right) d \xi d \eta
$$

which yields

$$
\begin{aligned}
q_{\text {modeled }}\left(k_{x}, k_{y}, \alpha, \beta, f\right)= & \frac{4 l_{x} l_{y}}{\left(1+\left(k_{x} \cos \alpha+k_{y} \sin \alpha-k_{\varphi} \cos (\beta-\alpha)\right)^{2} \cdot l_{x}^{2}\right)} \\
& \cdot \frac{1}{\left(1+\left(k_{y} \cos \alpha-k_{x} \sin \alpha-k_{\varphi} \sin (\beta-\alpha)\right)^{2} \cdot l_{y}^{2}\right)}
\end{aligned}
$$

A more detailed derivation of the Fourier transform can be found in the appendix. Values for the phase velocity $u_{\varphi}$ can be obtained from the model by Smol'Yakov. ${ }^{15}$ 


\section{Results}

In the following, a modeled illustration of both, the coherence pattern in the spatial domain, and of wavenumber spectra will be shown. For each sketch, experimental data will be presented in which such a modeled behavior is believed to occur. First, the modeled coherence pattern will be compared to experimental data and afterwards, two modeled wavenumber spectra will be compared with experimentally obtaind wavenumber spectra at different frequencies.

\section{V.A. Spatial Domain}

In figure 3 the modeled coherence pattern using $l_{x}=1.4 \mathrm{~m}$ and $l_{y}=0.2 \mathrm{~m}$ is shown. These values are arbitrarily chosen for good display of the model in the figure. The inclination angle was chose to be $\alpha=10^{\circ}$ and as a result, the rhombic shape representing the outline of the coherence pattern at a threshold of $\gamma_{\text {thresh }}=\exp (-1)$ is tilted upwards. The angle of the longtest straight line to fit into the rhombic shape is tilted relative to the array length axis shown as a dashed line at $\eta=0$. The rhombic shape results from the multiplicative approach for combining $l_{x}$ and $l_{y}$.

For the two evaluation frequencies chosen $(f=732 \mathrm{~Hz}$ in figure 4 and $f=2490 \mathrm{~Hz}$ in figure 5) the coherence pattern shows a similar inclination of the coherence pattern as displayed in the model. Such a deviation of the flow relative to the array orientation has been widely observed and the present analysis method has been presented in detail by Haxter\&Spehr. ${ }^{4}$ Note that the coherence pattern in the experimental data is larger at lower frequency than it is at higher frequency because the coherence lengths in both, streamwise and cross-stream direction are a function of frequency. The inclination does not differ greatly between the two frequencies chosen for evaluation.

\section{V.B. Wavenumber Domain}

A sketch of a wavenumber domain where both, the direction of the coherence pattern and the direction of phase propagation are similarly inclined relative to the array by an angle $\hat{\alpha}$ is shown in figure 6 . The angle from the origin of the spectrum to the center of the convective ridge is chosen similar to the last case of inclined coherence pattern in figure 3. In the modeled spectrum, a line from the origin of the spectrum intersects with the inclination of the convective ridge at an angle of $90^{\circ}$.

A similar characteristic is seen in the experimental data at a frequency of $f=2490 \mathrm{~Hz}$. The center of the convective ridge was definded as the center of the shape resulting from applying a $-5 \mathrm{~dB}$ threshold to the data.

When the angle of coherence pattern inclination and the angle of phase propagation differ, the convective ridge experiences a translational displacement in the wavenumber domain as seen in figure 8. Since the angle of coherence pattern inclination was not changed from figure 6 , the inclination of the convective ridge did not change. However, the position of the center of the convective ridge has move downwards and lies at an angle of $\beta=-2^{\circ}$ relative to the origin. In the experimental data, a very similar characteristic is observed at a frequency of $f=586 \mathrm{~Hz}$. While the convective ridge is clearly still inclined, its center is now shifted downwards and located very close to $k_{y}=0$.

\section{V.C. Dependence on frequency}

Exemplary results for the methods under consideration were shown for discrete frequencies above. The frequency-dependence of the angles resulting from different methods is shown in figure 10. Both, the angle obtained from the tilt of the coherence pattern and the angle from the inclination of the convective ridge show a quasi-constant offset of approximately $12^{\circ}$ to $15^{\circ}$ at frequencies below $f=2800 \mathrm{~Hz}$. Smaller discrepancies between these two curves can be observed and they are caused most likely due to the lack of resolution of transducers with a coherence value above the chosen threshold of exp -1. Sharp peaks at multiples of $400 \mathrm{~Hz}$ are caused by electrical noise on the signals. At higher frequencies, the angle from the tilt of the coherence pattern yields an angle of $0^{\circ}$ for all frequencies. This too is a result of the lack of transducer spacings entering the analysis, because the coherence length is to short to create a coherence pattern large enough to evaluate at this threshold.

The angle resulting from the location of the center of the convective ridge shows a different characteristic at frequencies below $f=1400 \mathrm{~Hz}$. The angle increases linearly from approximately $-30^{\circ}$ at $200 \mathrm{~Hz}$ to $10^{\circ}$ 


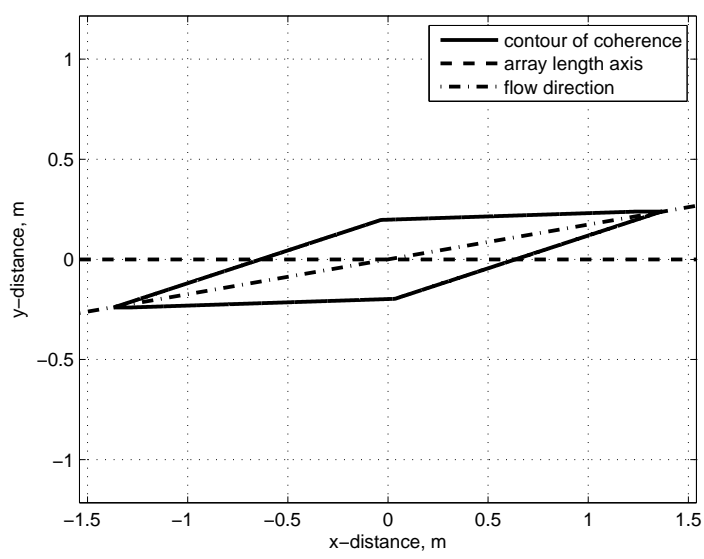

Figure 3. Sketch: angle determined from coherence: in this example, the flow direction is tilted $10^{\circ}$ relative to the length axis.

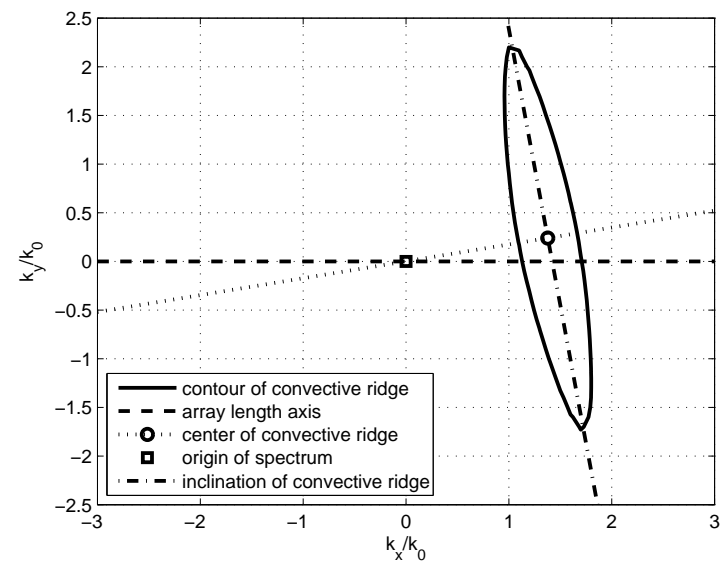

Figure 6. Sketch: angle determined from wavenumber spectrum: in this example, the flow direction is tilted $10^{\circ}$ relative to the length axis. Both, phase velocity and flow direction are aligned which results in an angle of $90^{\circ}$ between the inclination of the convective ridge and the line from the center of the convective ridge to the origin.

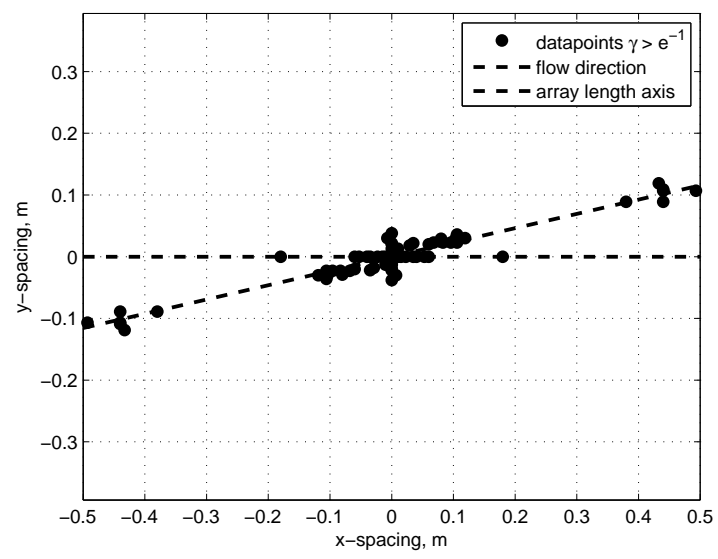

Figure 4. Experimental data: angle determined from coherence at $f=537 \mathrm{~Hz}$.

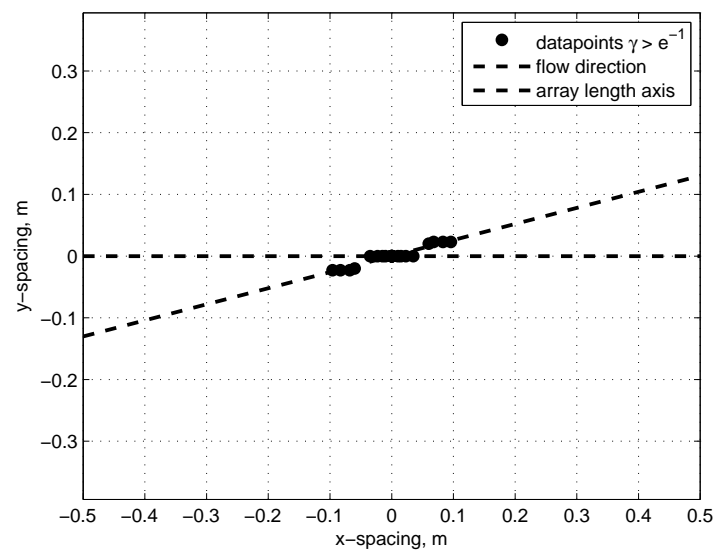

Figure 5. Experimental data: angle determined from coherence at $f=2490 \mathrm{~Hz}$

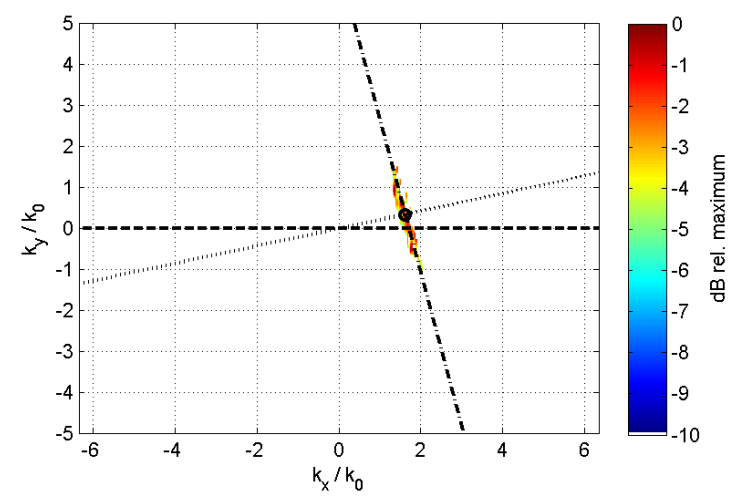

Figure 7. Wavenumber spectrum from experimental data at $f=2490 \mathrm{~Hz}$ : the convective ridge is inclined and the the center of the convective ridge is tilted upwards as well. Both, phase velocity and flow direction are aligned which results in an angle of $90^{\circ}$ between the inclination of the convective ridge and the line from the center of the convective ridge to the origin. 


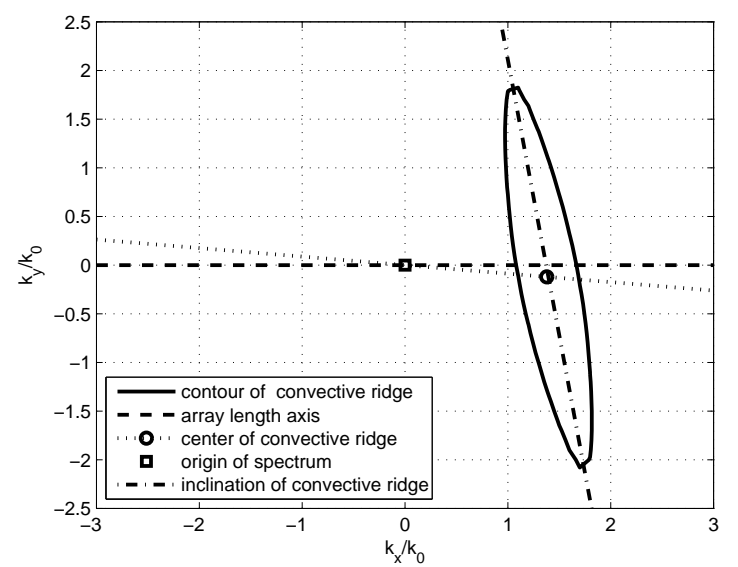

Figure 8. Sketch: angle determined from wavenumber spectrum: in this example, the flow direction is tilted $10^{\circ}$ relative to the length axis. Phase velocity and flow direction are not aligned which results in the angle between the inclination of the convective ridge and the line from the center of the convective ridge to the origin to differ from $90^{\circ}$.

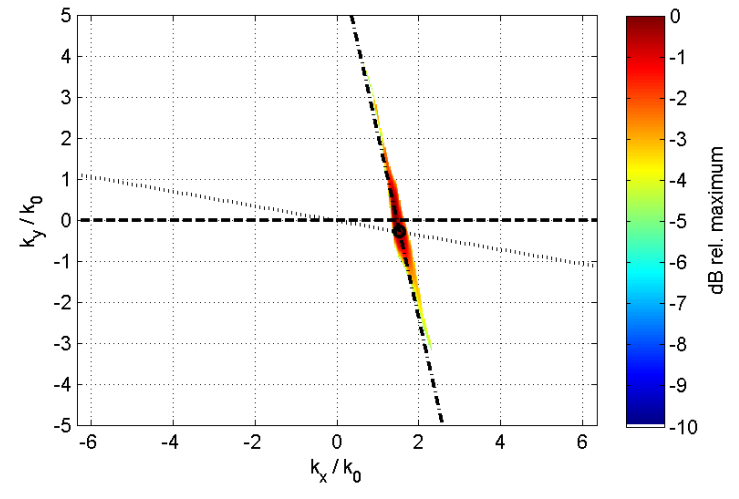

Figure 9. Wavenumber spectrum from experimental data at $f=586 \mathrm{~Hz}$ : the convective ridge is inclined while the center of the convective ridge is located approximately at $k_{y}=0$. Phase velocity and flow direction are not aligned which results in the angle between the inclination of the convective ridge and the line from the center of the convective ridge to the origin to differ from $90^{\circ}$.

at $1400 \mathrm{~Hz}$. Above this frequency, the angle resulting from the center and the angle resulting from the inclination of the convective ridge show a similar behavior.

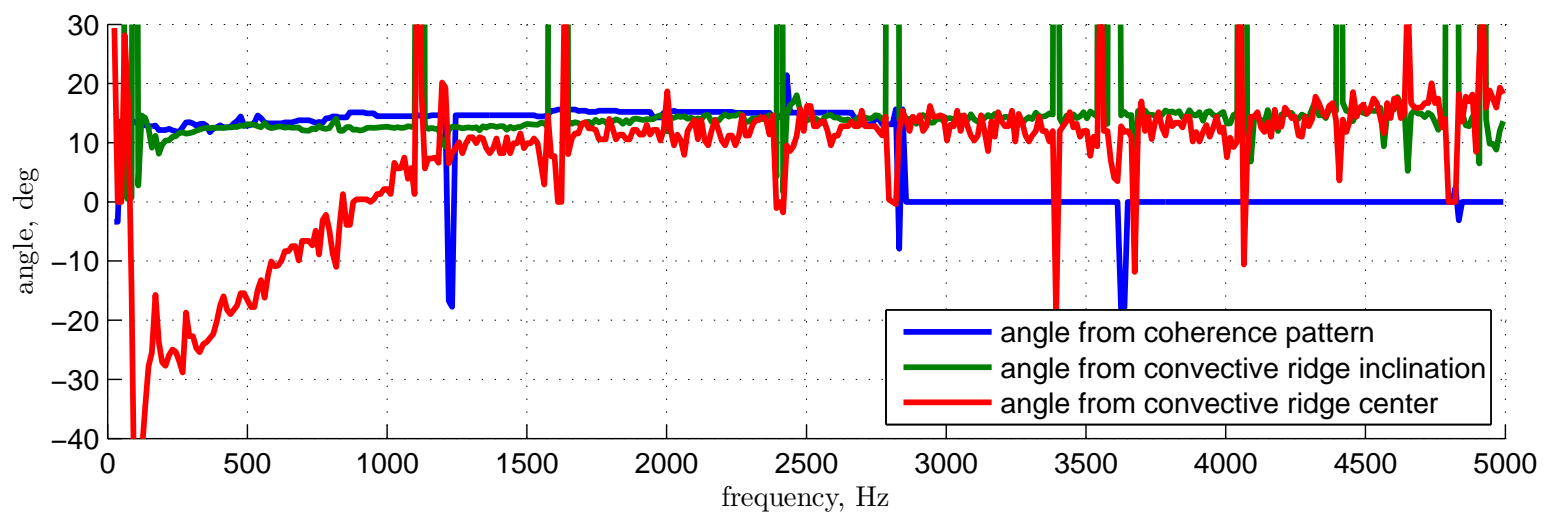

Figure 10. Angles resulting from the three different evaluation methods

\section{Discussion}

To the authors' knowledge, a difference between the inclination angle and the position of the convective ridge has not been reported so far. The approach taken in discussing the findings is therefore to ask: what are the individual causes for each, the inclination of the convective ridge and coherence pattern, as well as for the position of the center of the convective ridge? What are the physical reasons for them to be separated?

Furthermore, the effect of separate angles is frequency-dependent as shown in figure 10: while the angle difference is very pronounced at low frequencies, this difference disappears as the frequency increases and at high frequencies both, the flow direction from coherence pattern and from the inclination of the convective ridge, and the angle to the center of the convective ridge are aligned.

High-frequent pressure fluctuations can be caused by both small-scale structures propagating slowly over the array and by larger-scale structures propagating quickly over the array. However, looking at the coherence lengths of turbulent pressure fluctuations and that the average distance over which a turbulent structure 'lives' is approximately $6 \lambda$ (with $\lambda$ being the diameter) it is not unfeasible that large-scale structures at least 
dominate the power spectrum at the lower end of the frequency spectrum and small scale structures at the upper end of the frequency spectrum. For the following discussion it is therefore assumed that small-scale structures are connected with high frequencies.

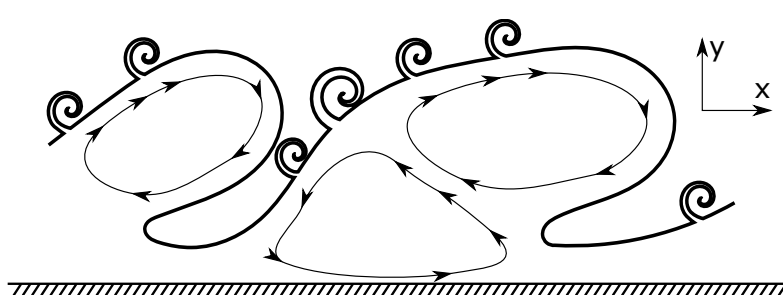

Figure 11. Sketch of the intermittent turbulent boundary layer with large scale motions and "typical eddies". (based on reference 16). The idealized rotation axis at the intersecting plane shown is perpendicular to the flow-direction.

If we continue this thought, then it appears as if small-scale vortices at high frequencies do not show off the angle discrepancy while, while vortices at lower frequencies do. A separation of the flow into two different "families of motion" was proposed by Falco $^{16}$ who analyzed the Lagrangian motion of turbulent structures in a boundary layer. In his work he distinguishes between a large-scale motion of the size of the boundary layer thickness and so-called "typical eddies" which are small vortices superimposed on the rim of the large-scale structures. At low Reynolds numbers, the size of the "typical eddies" is in the order of the boundary layer thickness. As the Reynolds number increases, the relative size of the typical eddies decreases. In the structural model proposed by Falco, the typical eddies move about on the rim of the large-scale structure in a direction depending on their initial position. This is shown in figure 11.

The model proposed by Falco is just two-dimensional and the rotation axis of the large-scale-motion is in cross-stream direction. However, Falco mentions that the evaluation was affected by large-scale-motions moving in and out of the evaluation plane under consideration, thereby indicating that a three-dimensional movement is possible. If the idea of a two-dimensionality of the large-scale motion is discarded in favor of a tilt about the wall-normal axis, such a turbulent structure might cause the effects observed.

It is known from boundary layers on swept wings that the velocity profile can take three-dimensional characteristics. ${ }^{17}$ A sketch of this is shown in figure 12. The outer layer of the boundary layer and the nearwall part of the boundary layer are convected into different directions due to a pressure-induced crossflow component. If different frequency regions of the spectrum are associated with vortices at different distances to the wall, such a crossflow characteristic could explain the difference in flow direction at different frequencies.

(a)

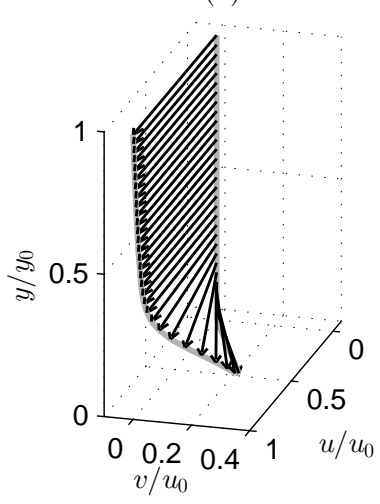

(b)

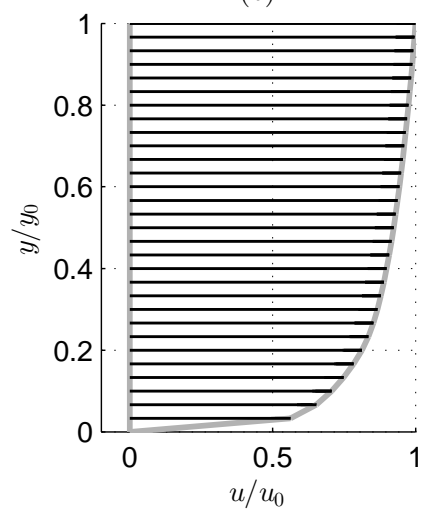

(c)

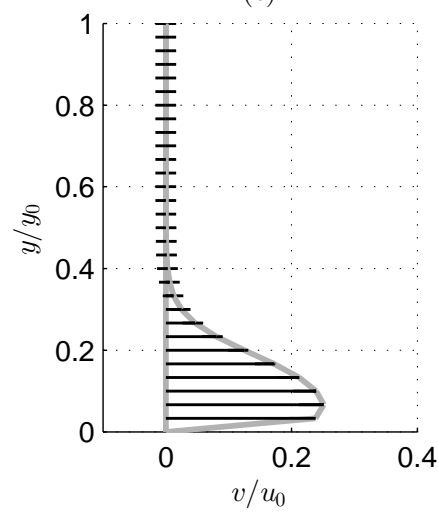

Figure 12. Sketch of a three-dimensional boundary layer due to crossflow: close to the wall, the flow is deviated from the flow direction.

The question remains how the phase velocity - responsible for the position of the convective ridge -and the convective velocity - responsible for the inclination of the convective ridge and the tilt of the coherence pattern - show separate directions at low frequencies. If a crossflow component was present during the measurement, it is possible that this crossflow-component was on the one hand large enough to affect nearwall structures and locally change their propagation direction, but on the other hand not large enough to totally affect the large-scale motion. In other words: the rotation of the large-scale motion would not just be as indicated in figure 11, but would have another component rotating about the length axis and resultin in a large scale 3D motion. Such a rotation would propagate near-wall structures in a different direction than 
the large scale motion.

At low frequencies, the coherence length is longer than at higher frequencies as long as the vortex size is not limited by the boundary layer thickness. ${ }^{4,11}$ The lifelength-distance and the boundary layer limitation indicate that the coherent pressure fluctuations at low frequencies are caused by large-scale motion on the scale of the boundary layer thickness. The occurrence of distortion due to a crossflow component at similar frequency suggests such a behavior. At large-scale distances - where coherent pressure from the large-scale motion is expected - the overall and undisturbed flow direction is predominant. At small-scale distance where coherent pressure form the small-scale motion is expected - the disturbed flow direction is predominant.

The importance of this characteristic lies in the prediction of structural vibration. As the position of the convective ridge determines which structural modes are to be excited, a translation of the center position of the convective ridge - including its maximum position - can greatly affect the prediction.

\section{Summary}

Flight test data was analyzed with the aim to obtain wavenumber spectra of surface pressure fluctuations. Typical features of such wavenumber spectra for a turbulent boundary layer flow were be identified. Dependent on frequency however, a translation of the convective ridge was noted, resulting in a deviation of the convective ridge inclination from the angle to the convective wavenumber. The occurrence of a crossflow on the fuselage, induced by the displacement of flow in the vicinity of the wing, is believed to be the cause for this effect. The crossflow yields a rotated flow field in the vicinity of the wall, leaving small scale structures unchanged, but distorts the large-scale motion in the boundary layer. The assumed cause shall be further investigated.

\section{References}

\footnotetext{
${ }^{1}$ Haxter, S. and Spehr, C., "Comparison of model predictions for coherence length and convective velocity to in-flight measurements at cruise conditions," Journal of Sound and Vibration;, Vol. accepted for publication, 2016.

${ }^{2}$ Rizzi, S. A., Rackl, R. G., and Andrianov, E. V., "Flight test measurements from the Tu-144LL structure/cabin noise follow-on experiment," 2000.

${ }^{3}$ Palumbo, D., "Determining correlation and coherence lengths in turbulent boundary layer flight data," Journal of Sound and Vibration, Vol. 331, No. 16, 2012, pp. 3721-3737.

${ }^{4}$ Haxter, S. and Spehr, C., "Comparison of model predictions for coherence length to in-flight measurements at cruise conditions," Journal of Sound and Vibration, Accepted for publication.

${ }^{5}$ Abraham, B. M. and Keith, W. L., "Direct measurements of turbulent boundary layer wall pressure wavenumberfrequency spectra," Journal of Fluids Engineering, Vol. 120, No. 1, 1998, pp. 29-39.

${ }^{6}$ Haxter, S., Spehr, C., Hartmann, M., Ocker, J., Tokuno, H., and Wickern, G., "Improving the performance of aeroacoustic measurements beneath a turbulent boundary layer in a wake flow," AIAA Aviation. American Institute of Aeronautics and Astronautics, 2014.

${ }^{7}$ Bull, M., "Wall-pressure Fluctuations Beneath Turbulent Boundary Layers: Some Reflections On Forty Years Of Research," Journal of Sound and Vibration, Vol. vol. 190, part 3, 1996, pp. pp. 299-315.

${ }^{8}$ Ehrenfried, K. and Koop, L., "Experimental study of pressure fluctuations beneath a compressible turbulent boundary layer," Paper 2008-2800, 14th AIAA/CEAS Aeroacoustics Conference, Vancouver, British Columbia, Canada, 2008.

${ }^{9}$ Haxter, S., "Extended Version: Improving the DAMAS 2 Results for Wavenumber-Space Beamforming," Paper BeBeC2016-D8; Proceedings of the 7th Berlin Beamforming Conference, February 2016.

${ }^{10}$ Corcos, G. M., "Resolution of Pressure in Turbulence," The Journal of the Acoustical Society of America, Vol. vol. 35, No. 2, 1963, pp. 192-199.

${ }^{11}$ Efimtsov, M., "Characteristics of the Field of Turbulent Pressures at the Wall of a Boundary Layer," Sov. Phys. Acoust., Vol. 28, 1982, pp. No. 4.

${ }^{12}$ Robertson, J., "Prediction of in-flight fluctuating pressure environments including protuberance induced flow," Tech. Rep. WR 71-10, Wyle Laboratories Research Staff, 1971.

${ }^{13}$ Harris, F. J., "On the Use of Windows for Harmonic Analysis with the Discrete Fourier Transform," Proceedings of the IEEE, Vol. vol. 66, No. 1, 1978.

${ }^{14}$ Digital Image Processing, Tata McGraw Hill Education, 2011.

${ }^{15}$ Smol'yakov, A. V., "A New Model for the Cross Spectrum and WavenumberFrequency Spectrum of Turbulent Pressure Fluctuations in a Boundary Layer," Vol. 52, No. 3, 2006, pp. 331-337.

${ }^{16}$ Falco, R., "Coherent motions in the outer region of turbulent boundary layers," Physics of Fluids (1958-1988), Vol. 20, No. 10, 1977, pp. S124-S132.

${ }^{17}$ Saric, W. S., Reed, H. L., and White, E. B., "Stability and transition of three-dimensional boundary layers," Annual Review of Fluid Mechanics, Vol. 35, No. 1, 2003, pp. 413-440.
} 


\section{Appendix}

The Fourier transform of the tilted coherence pattern with the shifted propagation angle will be derived in the following. Starting point is the Fourier transform of a function $\gamma(\xi, \eta, \alpha)$ representing the coherence decay and a function $\varphi(\xi, \eta, \beta)$ representing the propagation. $\xi$ and $\eta$ are the transducer separations in $\mathrm{x}$ - and $\mathrm{y}$-direction respectively. $\alpha$ is the tilt angle of the coherence pattern and similarly the angle of the convective ridge. $\beta$ is the propagation direction and similarly the angle from the origin of the wavenumber spectrum to the center of the convective ridge. The transformation is

$$
\Phi\left(k_{x}, k_{y}, \alpha, \beta, f\right)=\frac{1}{(2 \pi)^{2}} \int_{-\infty}^{\infty} \int_{-\infty}^{\infty} \gamma(\xi, \eta, \alpha, f) \cdot \varphi(\xi, \eta, \beta, f) \cdot \exp \left(-i\left(k_{x} \xi+k_{y} \eta\right)\right) d \xi d \eta
$$

with

$$
\gamma(\xi, \eta, \alpha, f)=\exp \left(-\frac{|\xi \cos \alpha+\eta \sin \alpha|}{l_{x}(f)}-\frac{|-\xi \sin \alpha+\eta \cos \alpha|}{l_{y}(f)}\right)
$$

and

$$
\varphi(\xi, \eta, \beta, f)=\exp \left(-i \cdot k_{\varphi}(\xi \cos \beta+\eta \sin \beta)\right) .
$$

Equation (25) is written in terms of the aircraft coordinate system. It can be transformed to the coordinate system of the tilted coherence pattern via

$$
\left(\begin{array}{l}
u \\
v
\end{array}\right)=\left(\begin{array}{cc}
\cos \alpha & -\sin \alpha \\
\sin \alpha & \cos \alpha
\end{array}\right)\left(\begin{array}{l}
\xi \\
\eta
\end{array}\right)
$$

The Jacobian of the transformation in equation (26) is 1 , so that.

$$
d \xi d \eta=\left|\operatorname{det} \frac{\partial(\xi, \eta)}{\partial(u, v)}\right| d u d v=d u d v
$$

The Fourier transform is then written as

$$
\begin{aligned}
\Phi\left(k_{x}, k_{y}, \alpha, \beta\right)=\frac{1}{(2 \pi)^{2}} \int_{-\infty}^{\infty} \int_{-\infty}^{\infty} & \exp \left(-\frac{|u|}{l_{x}}-\frac{|v|}{l_{y}}\right) \\
\cdot & \exp \left(-i\left(k_{x}(u \cos \alpha-v \sin \alpha) k_{y}(u \sin \alpha+v \cos \alpha)\right)\right) \\
\cdot & \exp \left(-i k_{\varphi}(\cos \beta(u \cos \alpha-v \sin \alpha)+\sin \beta(u \sin \alpha+v \cos \alpha))\right)
\end{aligned}
$$

This can be split into separate integrals:

$$
\begin{aligned}
\Phi\left(k_{x}, k_{y}, \alpha, \beta\right)=\frac{1}{(2 \pi)^{2}} & \int_{-\infty}^{\infty} \exp \left(-\frac{|u|}{l_{x}}-i u\left(k_{x} \cos \alpha+k_{y} \sin \alpha+k_{\varphi}(\cos \beta \cos \alpha+\sin \beta \sin \alpha)\right)\right) d u \\
& \int_{-\infty}^{\infty} \exp \left(-\frac{|v|}{l_{y}}-i v\left(-k_{x} \sin \alpha+k_{y} \cos \alpha+k_{\varphi}(-\cos \beta \sin \alpha+\sin \beta \cos \alpha)\right)\right) d v
\end{aligned}
$$

which can be reduced to

$$
\Phi\left(k_{x}, k_{y}, \alpha, \beta\right)=\frac{1}{(2 \pi)^{2}} \int_{-\infty}^{\infty} \exp \left(-\frac{|u|}{l_{x}}-i u p\right) d u \cdot \int_{-\infty}^{\infty} \exp \left(-\frac{|v|}{l_{y}}-i v q\right) d v .
$$

by using

$$
p=k_{x} \cos \alpha+k_{y} \sin \alpha+k_{\varphi} \cos (\beta-\alpha)
$$

and

$$
q=-k_{x} \sin \alpha+k_{y} \cos \alpha+k_{\varphi} \sin (\beta-\alpha)
$$


This can be evaluated by splitting up the ranges of integration

$$
\begin{aligned}
\Phi\left(k_{x}, k_{y}, \alpha, \beta\right)=\frac{1}{4 \pi^{2}} & \cdot\left(\int_{0}^{\infty} \exp \left(-u\left(\frac{1}{l_{x}}+i p\right)\right) d u+\int_{0}^{\infty} \exp \left(-u\left(\frac{1}{l_{x}}-i p\right)\right) d u\right) \\
& \left(\int_{0}^{\infty} \exp \left(-v\left(\frac{1}{l_{y}}+i q\right)\right) d v+\int_{0}^{\infty} \exp \left(-v\left(\frac{1}{l_{y}}-i q\right)\right) d v\right)
\end{aligned}
$$

This yields the Fourier transform

$$
\Phi\left(k_{x}, k_{y}, \alpha, \beta\right)=\frac{4 l_{x} l_{y}}{4 \pi^{2}\left(1+l_{x}^{2} p^{2}\right)\left(1+l_{y}^{2} q^{2}\right)}
$$

Substituting for $p$ and $q$ again yields

$$
\begin{aligned}
\Phi\left(k_{x}, k_{y}, \alpha, \beta\right)= & \frac{l_{x} l_{y}}{\pi^{2}} \\
& \cdot \frac{1}{1+l_{x}^{2}\left(k_{x} \cos \alpha+k_{y} \sin \alpha+k_{\varphi} \cos (\beta-\alpha)\right)^{2}} \\
& \cdot \frac{1}{1+l_{y}^{2}\left(-k_{x} \sin \alpha+k_{y} \cos \alpha+k_{\varphi} \sin (\beta-\alpha)\right)^{2}} .
\end{aligned}
$$

\section{Acknowledgments}

The authors wish to acknowledge the following institutions for their support:

- The Federal Ministry for Economic Affairs and Energy (BMWi) for the financial support of the flight test in the "SIMKAB" campaign as part of the aerospace research program (LuFO IV; Support code $20 \mathrm{~K} 0805 \mathrm{G})$.

- Airbus S.A.S. for the provided infrastructure and help on modifications of the flight test carrier.

- The DLR Institute of Aero-Elasticity, DLR Institute of Flight Systems, and the DLR Flight Experiment team for the help provided in the preparation and performance of the flight test. 